\title{
Evaluation of left ventricular systolic function revisited in septic shock
}

\author{
Xavier Repessé, ${ }^{1,2}$ Cyril Charron ${ }^{1,2}$ and Antoine Vieillard-Baron ${ }^{* 1,2}$ \\ See related research by Huang et al., http://ccforum.com/content/17/3/R96
}

\begin{abstract}
The meta-analysis of Huang and coworkers failed to find any evidence for a protective effect of a decreased left ventricular (LV) ejection fraction (EF). These results have to be interpreted with caution since in most studies included in the meta-analysis patients with LV systolic dysfunction received inotropic drugs. We have some arguments suggesting that such a treatment may improve macrocirculation and microcirculation and finally prognosis. This paper allows us to clarify the meaning of LV function in septic shock patients. In all experimental models of septic shock using the load-independent parameter of LV systolic function, LV contractility impairment, called septic cardiomyopathy, has been reported to be constant. However, LVEF reflects the coupling between LV contractility and LV afterload. A normal LVEF may be observed when the arterial tone is severely depressed, as in septic shock, despite seriously impaired intrinsic LV contractility. LV systolic function, evaluated using an echocardiograph or another device, is then more a reflection of arterial tone (and its correction) than of intrinsic LV contractility. As a consequence, the incidence of LV systolic dysfunction greatly depends on the time of the evaluation, reflecting the fact that, during resuscitation and treatment, vasoplegia and then LV afterload are corrected, thus unmasking septic cardiomyopathy. With these points in mind, we can revisit the results of Margaret Parker's original study: it is not that the patients with a low EF survived better, but rather that the other patients had an increased mortality due to persistent profound vasoplegia.
\end{abstract}

In the previous issue of Critical Care, Huang and colleagues try to answer the unresolved question of the prognostic value of sepsis-related cardiomyopathy [1]. Since Margaret Parker and colleagues originally reported in 1984 that $65 \%$ of patients had significant left ventricular (LV) systolic dysfunction in the early phase of sepsis associated with acute LV dilatation ( $>100 \%$ increase in size!) and that such patients had a better prognosis [2], various groups have failed to replicate these results, leading to confusion and controversy.

The article by Huang and colleagues is interesting because it reports a large meta-analysis including more than 700 septic patients available for LV analysis. The meta-analysis failed to find any evidence for a protective effect of a decreased LV ejection fraction (EF) [1]. Nevertheless, the nonindexed LV dimension was moderately higher among survivors than nonsurvivors [1]. These results have to be interpreted with caution since in most studies included in the meta-analysis patients with LV systolic dysfunction received inotropic drugs. In the study by Cariou and colleagues in 10 patients, most patients were infused with epinephrine or dobutamine [3]. In the study performed by our group in 68 patients, most patients received dobutamine [4]. We have some arguments suggesting that such a treatment may improve prognosis. Bouferrache and colleagues reported recently that dobutamine significantly improves the macrocirculation in patients with a low flow state who show a $40 \%$ increase in cardiac output despite normal venous oxygen saturation [5]. This improvement was sustained by a $50 \%$ increase in LVEF. Interestingly, such improvement leads to microcirculatory amelioration. De Backer and colleagues demonstrated that the proportion of functional capillaries was decreased in septic patients compared with volunteers [6] and that dobutamine, by inducing a $21 \%$ increase in cardiac output, led to a nearly complete reversal of such alterations [7]. In the study by Rivers and \footnotetext{
colleagues, demonstrating a better prognosis in the early goal-directed therapy group, close to $14 \%$ of patients received dobutamine in the first 6 hours in the interventional group versus $0.8 \%$ in the control group [8]. Finally, Rhodes and colleagues, Kumar and colleagues,

'Service de réanimation, Assistance Publique-Hôpitaux de Paris, Hôpital Ambroise Paré, 9 avenue Charles-de-Gaulle, 92100 Boulogne-Billancourt, France Full list of author information is available at the end of the article 
and Vallet and colleagues reported similar results - a huge decrease in mortality in septic patients who respond to dobutamine in terms of cardiac output [9-11].

More interestingly, however, the article by Huang and colleagues allows us to try to clarify the meaning of LV function in septic shock patients. A lot of confusion exists. In all experimental models of septic shock, LV contractility impairment - called septic cardiomyopathy - has been reported to be constant. In these studies, as in the study by Barraud and colleagues [12], intrinsic contractility is assessed using a parameter that is not dependent on load conditions; that is, systolic elastance. Unfortunately, this assessment requires the generation of pressure/volume loops, something difficult to achieve in human subjects at the bedside. This difficulty is why in clinical practice most intensivists use LV systolic function parameters that are for the most part dependent on load conditions. This dependency is the case for LVEF obtained using echocardiography.

More than 20 years ago, Robotham and colleagues nicely reiterated that LVEF reflects the coupling between LV contractility and LV afterload [13]. In other words, a normal LVEF may be observed when the arterial tone is severely depressed, despite seriously impaired intrinsic LV contractility. Everyone understands that it is crucial to remember this in septic shock, in which arterial tone is initially severely decreased. LV systolic function, evaluated using an echocardiograph or another device, is more a reflection of arterial tone (and its correction) than of intrinsic LV contractility. In a 1990 study, Jardin and colleagues elegantly showed that patients with a normal LVEF had a significantly lower systemic vascular resistance than patients with a low LVEF in whom resistance was corrected [14]. As shown in Figure 1, it is then easy to understand that the incidence of LV systolic dysfunction greatly depends on the time of the evaluation. This dependence only reflects the fact that, during resuscitation and treatment, vasoplegia and then LV afterload are corrected, thus unmasking septic cardiomyopathy.

With these points in mind, we can revisit the results of Margaret Parker and colleagues' study: it is not that the patients with a low EF survived better, but rather that the other patients had an increased mortality due to persistent profound vasoplegia. This was suggested by our group in a study where $100 \%$ of patients with a hyperkinetic state (LVEF $67 \pm 7 \%$ ) finally died, compared with 'only' $43 \%$ of patients with a hypokinetic state (LVEF $34 \pm 10 \%$ ) [15]. Weng and colleagues showed recently that high peak systolic velocity measured at the mitral annulus by tissue Doppler imaging might be associated with mortality in patients with septic shock, suggesting that profound vasoplegia inducing high contractility is linked to poor prognosis [16].

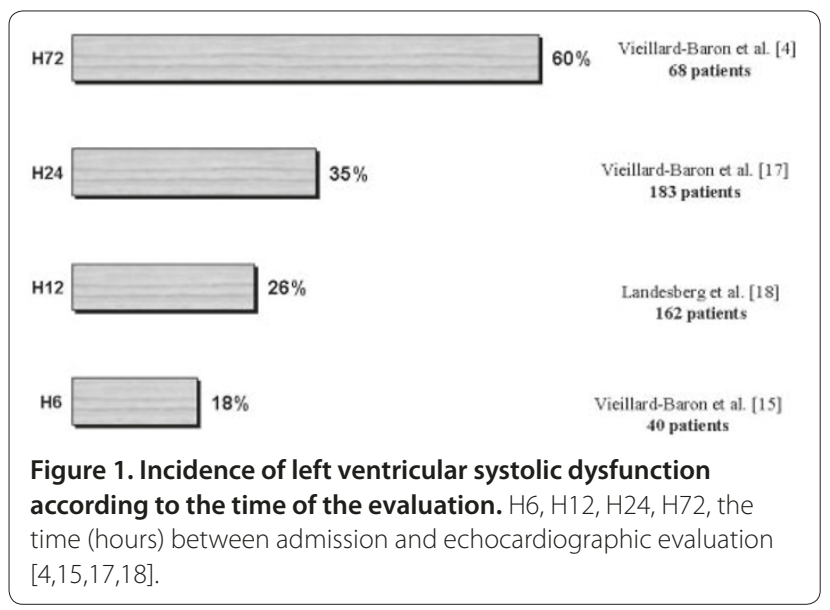

In conclusion, it will be very difficult to demonstrate that LV systolic dysfunction is associated with prognosis. Septic cardiomyopathy is constant and LV systolic function is more a reflection of the status of LV afterload. Rather, we have now to demonstrate what the best mortality-reducing strategy is when there is LV systolic dysfunction. Persistence of a hyperkinetic state is a warning signal suggesting that the septic process is not under control and that the patient has a high probability of dying.

Abbreviations

$E F$, ejection fraction; LV, left ventricular.

Competing interests

The authors declare that they have no competing interests.

Acknowledgements

Support was provided solely from institutional and/or departmental sources.

\section{Author details}

'Service de réanimation, Assistance Publique-Hôpitaux de Paris, Hôpital Ambroise Paré, 9 avenue Charles-de-Gaulle, 92100 Boulogne-Billancourt, France. ${ }^{2}$ Faculty of Medicine Paris Ile-de-France Ouest, University of Versailles Saint-Quentin en Yvelines, Saint-Quentin en Yvelines 78280, France.

Published: 4 July 2013

References

1. Huang SJ, Nalos M, McLean A: Is early ventricular dysfunction or dilatation associated with lower mortality rate in adult severe sepsis and septic shock? A meta-analysis. Crit Care 2013, 17:R96.

2. Parker MM, Shelhamer JH, Bacharach SL, Green M, Natanson C, FrederickT, Damske B, Parrillo J: Profound but reversible myocardial depression in patients with septic shock. Ann Intern Med 1984, 100:483-490.

3. Cariou A, Pinsky MR, Monchi M, Laurent I, Vinsonneau C, Chiche JD, Charpentier J, Dhainaut JF: Is myocardial adrenergic responsiveness depressed in human septic shock? Intensive Care Med 2008, 34:917-922.

4. Vieillard-Baron A, Caille V, Charron C, Belliard G, Page B, Jardin F: Actual incidence of global left ventricular hypokinesia in adult septic shock. Crit Care Med 2008, 36:1701-1706.

5. Bouferrache K, Amiel JB, Chimot L, Caille V, Charron C, Vignon P, VieillardBaron A: Initial resuscitation guided by the Surviving Sepsis Campaign recommendations and early echocardiographic assessment of hemodynamics in intensive care unit septic patients: a pilot study. Crit Care Med 2012, 40:2821-2827.

6. De Backer D, Creteur J, Preiser JC, Dubois MJ, Vincent JL: Microvascular blood 
flow is altered in patients with sepsis. Am J Respir Crit Care Med 2002, 166:98-104.

7. De Backer D, Creteur J, Dubois MJ, Sakr Y, Koch M, Verdant C, Vincent JL: The effects of dobutamine on microcirculation alterations in patients with septic shock are independent of its systemic effects. Crit Care Med 2006, 34:403-408.

8. Rivers E, Nguyen B, Havstad S, Ressler J, Muzzin A, Knoblich B, Peterson E, Tomlanovitch M; Early-Goal-Directed Therapy Collaborative Group: Early-goal directed therapy in the treatment of severe sepsis and septic shock. N Engl J Med 2001, 345:1368-1377.

9. Rhodes A, Lamb FJ, Malagon I, Newman PJ, Grounds RM, Benett D: A prospective study of the use of a dobutamine stress test to identify outcome in patients with sepsis, severe sepsis, or septic shock. Crit Care Med 1999, 27:2361-2366.

10. Kumar A, Schupp E, Bunnell E, Ali A, Milcarek B, Parrillo JE: Cardiovascular response to dobutamine stress predicts outcome in severe sepsis and septic shock. Crit Care 2008, 12:R35.

11. Vallet B, Curtis SE, Chopin C: Prognostic value of the dobutamine test in patients with sepsis syndrome. Crit Care Med 1995, 23:415-416.

12. Barraud D, Faivre V, Damy T, Welschbilling S, Gayat E, Heymes C, Payen D, Shah AM, Mebazaa A: Levosimendan restores both systolic and diastolic cardiac performance in lipopolysaccharide-treated rabbits: comparison with dobutamine and milrinone. Crit Care Med 2007, 35:1376-1382.
13. Robotham JL, Takata M, Berman M, Harasawa Y: Ejection fraction revisited. Anesthesiology 1991, 74:172-183.

14. Jardin F, Brun-Ney D, Auvert B, Beauchet A, Bourdarias JP: Sepsis-related cardiogenic shock. Crit Care Med 1990, 18:1055-1060.

15. Vieillard-Baron A, Schmidt JM, Beauchet A, Augarde R, Prin S, Page B, Jardin F: Early preload adaptation in septic shock? A transesophageal echocardiographic study. Anesthesiology 2001, 94:400-406.

16. Weng L, Liu YT, Du B, Zhou JF, Guo XX, Peng JM, Hu XY, Zhang SY, Fang Q, Zhu WL: The prognostic value of left ventricular systolic function measured by tissue Doppler imaging in septic shock. Crit Care 2012, 16:R71-R79.

17. Vieillard-Baron A, Prin S, Chergui K, Dubourg O, Jardin F: Hemodynamic instability in sepsis: bedside assessment by Doppler echocardiography. Am J Respir Crit Care Med 2003, 168:1270-1276.

18. Landesberg G, Gilon D, Meroz Y, Georgieva M, Levin PD, Goodman S, Avidan A, Beeri R, Weissman C, Jaffe AS, Sprung CL: Diastolic dysfunction and mortality in severe sepsis and septic shock. Eur Heart J 2012, 33:895-903.

doi:10.1186/cc12755

Cite this article as: Repessé $X$, et al.: Evaluation of left ventricular systolic function revisited in septic shock. Critical Care 2013, 17:164. 
